\title{
Contrast-enhanced voiding
} urosonography (CEVUS) as a novel technique for evaluation in a case of male urethral diverticulum

\author{
Shabnam Bhandari Grover, Sayantan Patra, Hemal Grover ${ }^{1}$, Anup Kumar² \\ Department of Radiology and Imaging, Vardhman Mahavir Medical College and Safdarjung Hospital, 'Department of Radiology, \\ Icahn School of Medicine at Mount Sinai West, New York, USA, ${ }^{2}$ Department of Urology and Renal Transplant, Vardhman \\ Mahavir Medical College and Safdarjung Hospital, New Delhi, India. \\ Correspondence: Dr. Shabnam Bhandari Grover, E-81, Kalkaji, New Delhi - 110 019, India. E-mail: shabnamgrover@yahoo.com
}

\begin{abstract}
Male urethral diverticulum is an uncommon entity, the abnormality being more frequently encountered in females. The pathology may be congenital or acquired and the more frequent acquired type usually occurs following trauma. Afflicted patients usually lack specific symptoms, although in a few instances, symptoms of lower urinary tract obstruction, calculi, or infection may prevail. Imaging investigations utilizing a composite Retrograde urethrography (RGU) - Voiding cystourethrography (VCUG) protocol are accepted as standard approach and ultrasound is considered a secondary supplementary investigation. However, recent literature reports the utility of contrast-enhanced ultrasound (CEUS) as a novel technique in the evaluation of urinary bladder and urethra, for vesico-ureteric reflux (VUR) in children and for urethral diverticula in women. We report a case of acquired post-traumatic urethral diverticulum in an adult male patient and document a relatively unexplored novel application of contrast enhanced voiding uro-sonography (CEVUS) for the evaluation of this malady.
\end{abstract}

Key words: Contrast-enhanced ultrasound (CEUS); contrast enhanced voiding urosonography (CEVUS); male urethral diverticulum; post-traumatic; RGU-VCUG.

\section{Introduction}

Male urethral diverticulum is a rare entity, the abnormality being more frequently encountered in females than in males. It refers to an abnormal outpouching from the urethral wall, into the adjacent fascia, which is in continuity with the urethral lumen $\cdot^{[1-4]}$ Afflicted patients usually lack specific symptoms, although in a few, symptoms due to dribbling, lower urinary tract obstruction, calculi, and infection may prevail. Imaging investigations reveal the diagnosis in

\begin{tabular}{|c|c|}
\hline \multicolumn{2}{|c|}{ Access this article online } \\
\hline Quick Response Code: & \\
\hline & $\begin{array}{l}\text { Website: } \\
\text { www.ijri.org }\end{array}$ \\
\hline & $\begin{array}{l}\text { DOI: } \\
\text { 10.4103/ijri.IJRI_50_20 }\end{array}$ \\
\hline
\end{tabular}

asymptomatic, but clinically suspected cases and confirm it in symptomatic patients. ${ }^{[2-5]}$ The standard imaging protocol is a composite Retrograde urethrography (RGU) - Voiding cysto-urethrography (VCUG) examination. ${ }^{[1,4-7]}$ Recent literature reports the utility of contrast enhanced ultrasound (CEUS) as a new technique for evaluation of urinary

This is an open access journal, and articles are distributed under the terms of the Creative Commons Attribution-NonCommercial-ShareAlike 4.0 License, which allows others to remix, tweak, and build upon the work non-commercially, as long as appropriate credit is given and the new creations are licensed under the identical terms.

For reprints contact: WKHLRPMedknow_reprints@wolterskluwer.com

Cite this article as: Grover SB, Patra S, Grover H, Kumar A. Contrast-enhanced voiding urosonography (CEVUS) as a novel technique for evaluation in a case of male urethral diverticulum. Indian J Radiol Imaging 2020;30:409-14.

Received: 08-Feb-2020 Revised: 09-Apr-2020 Accepted: 14-Jun-2020 Published: 15-Oct-2020 
bladder, urethra, urethral diverticula, and vesico-ureteric reflux (VUR) in children and for urethral diverticula in women. ${ }^{[8-11]}$ We report a relatively unexplored application of CEVUS for the evaluation in a case of adult male urethral diverticulum and emphasize its unique capability for real-time dynamic evaluation.

\section{Case Report}

A 45-year-old male presented with intermittent history of pyrexia, right lumbar pain, burning micturition, and urinary retention for 6 months. The patient was on interim treatment from an outside hospital with a nephrostomy drainage and antibiotics in their preparation for a right sided nephrectomy. The same had been planned for a diagnosis of post-obstructive atrophy and chronic right pyelonephritis, caused by a pelvi-ureteric junction (PUJ) calculus. The medical records did not show any specific management for episodes of urinary retention. Laboratory investigations from the same hospital showed neutrophilic leucocytosis in the hemogram and Klebsiella pneumoniae in urine culture. Ultrasound (US) kidney-ureter-bladder region and a computed tomography (CT) scan had been performed and reported as PUJ calculus on the right side complicated with post-obstructive atrophy and pyelonephritis in the right kidney.

Clinical evaluation at our hospital elicited a history of childhood perineal trauma with multiple attempts at urinary bladder catheterisation for urinary retention, at that time. Currently, routine laboratory investigations including hemogram and renal function tests were found to be within normal limits, whereas culture from nephrostomy drainage and bladder urine showed significant growth of Klebsiella nephrostomy pneumoniae (more than $10^{5}$ CFUs).

Imaging investigations were initiated with abdominal radiographs and US kidney-ureter-bladder region (US KUB). These were followed by fluoroscopic RGU-VCUG, CT urography, and Tc 99 DMSA scan, all performed using standard protocol. Abdominal radiograph showed a calculus in the right renal fossa with nephrostomy tube in situ. US KUB revealed a small right kidney, with PUJ calculus causing hydronephrosis, nephrostomy tube in situ along with changes of chronic obstruction in the urinary bladder and a diverticulum above the trigone [Figure 1]. RGU study revealed a stricture at the distal penile urethra with a large smooth-walled diverticulum located at the peno-bulbar region [Figure 2]. Another stricture was observed at the bulbo-membranous junction. VCUG additionally showed changes of chronic bladder obstruction and the urethral diverticulum [Figure 2]. There was mild dilatation in the posterior urethra, but no evidence of VUR [Figure 2]. CT urography demonstrated delayed excretion in a shrunken right kidney which had features of pyelonephritis with right ureteritis [Figures 3 and 4]. Tc-99 DMSA renal scintigraphy showed a small-sized right kidney, with delayed excretion and differential function of right kidney was found to be $30 \%$ of the total [Figure 4]. The imaging diagnosis arrived at was: right PUJ calculus, with right kidney post-obstructive
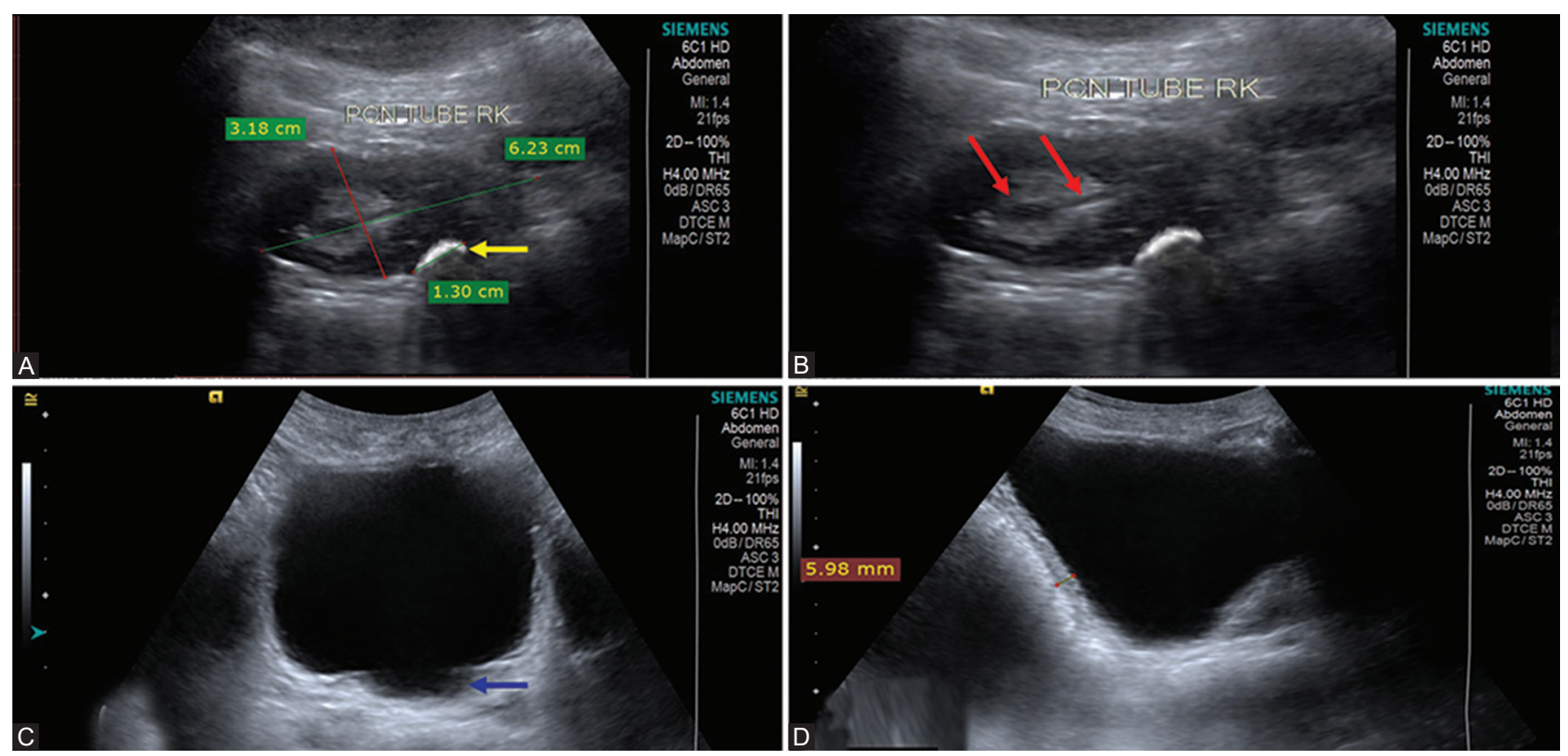

Figure 1 (A-D): Gray-scale ultrasound (US) of the KUB region in the 45-year-old male, diagnosed case of right PUJ calculus, complicated by post-obstructive atrophy in right kidney; who also had intermittent urinary obstruction - (A) shows small right kidney, measuring $6.23 \mathrm{~cm}$ in length, with a right PUJ calculus (yellow arrow), measuring $1.3 \mathrm{~cm}$; (B) shows nephrostomy drainage tube in situ (red arrows); (C) shows a urinary bladder diverticulum seen in the posterior wall (blue arrow); (D) shows that the urinary bladder wall is thickened and irregular, measuring 5.98 $\mathrm{mm}$ in thickness. 
atrophy with pyelonephritis, with compromised right renal excretion, with right ureteritis, with urethral strictures, and a urethral diverticulum, the latter two leading to chronic urinary bladder obstruction.

For further evaluation of the peno-scrotal soft tissues surrounding the urethral diverticulum, a CEVUS study was performed. The patient was first catheterized and a homogenized diluted US contrast agent solution was instilled into the urinary bladder, under US guidance, until complete filling was attained. The contrast solution was prepared by combining $1 \mathrm{ml}$ of reconstituted Sonovue $^{\mathrm{TM}}$ (Bracco, Milan, Italy) with $0.9 \%$ of normal saline and the volume made up to $500 \mathrm{ml}$. US examination was performed in supine position using a Siemens (Siemens, Munich, Germany) US machine, model Acuson S-3000, configured with contrast capabilities. Split screen contrast mode and low mechanical index (MI) settings were chosen for the study. Scans of bladder region and the urethra were acquired followed by scans of the renal region both during stationary and voiding phases, using appropriate probes relevant to the region/s being examined. CEVUS
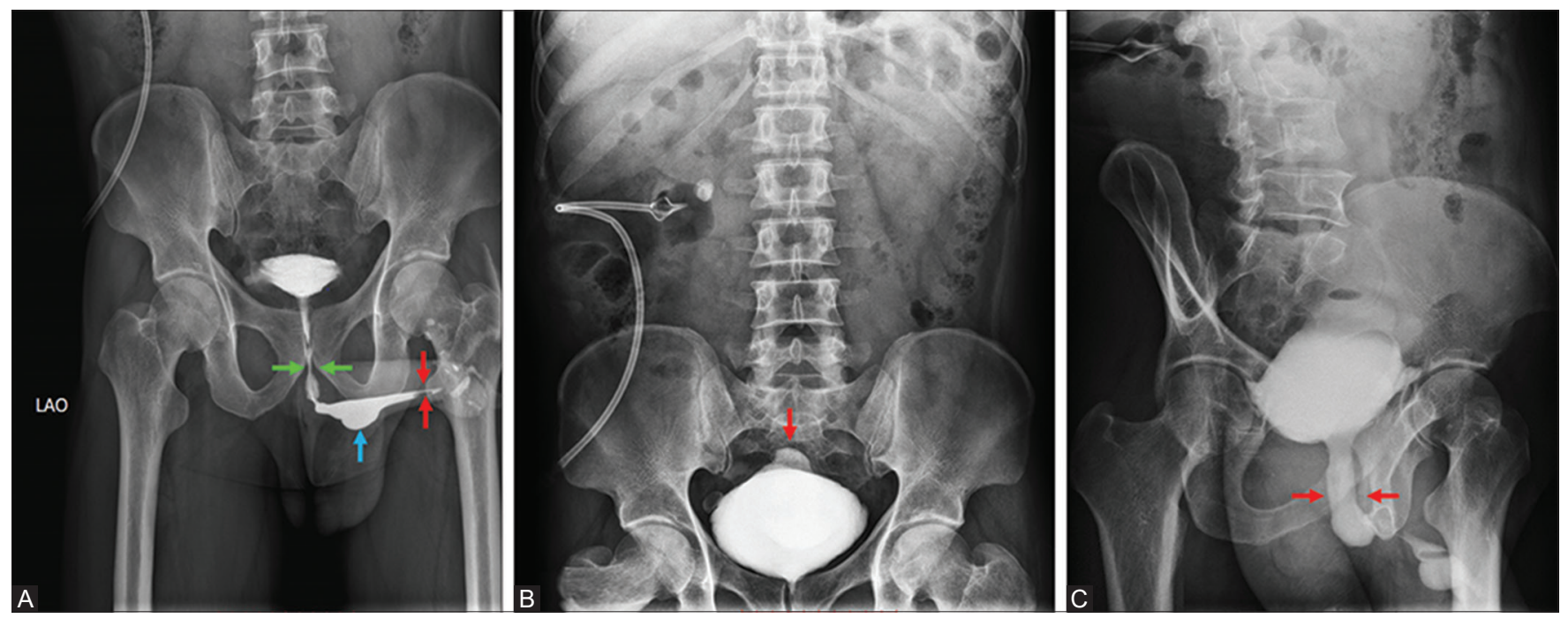

Figure 2 (A-C): RGU-VCUG examination in the 45-year-old male, diagnosed case of right PUJ calculus, complicated by post-obstructive atrophy in right kidney; who also had intermittent urinary obstruction, (A) shows strictures at the bulbar urethra (green arrows) and distal penile urethra (red arrows) with a large diverticulum at peno-bulbar junction (blue arrow); (B) full bladder phase of VCUG shows multiple sacculations and a large posterior diverticulum (red arrow); (C) voiding phase VCUG shows dilatation of posterior urethra (red arrows), there is no evidence of VUR.
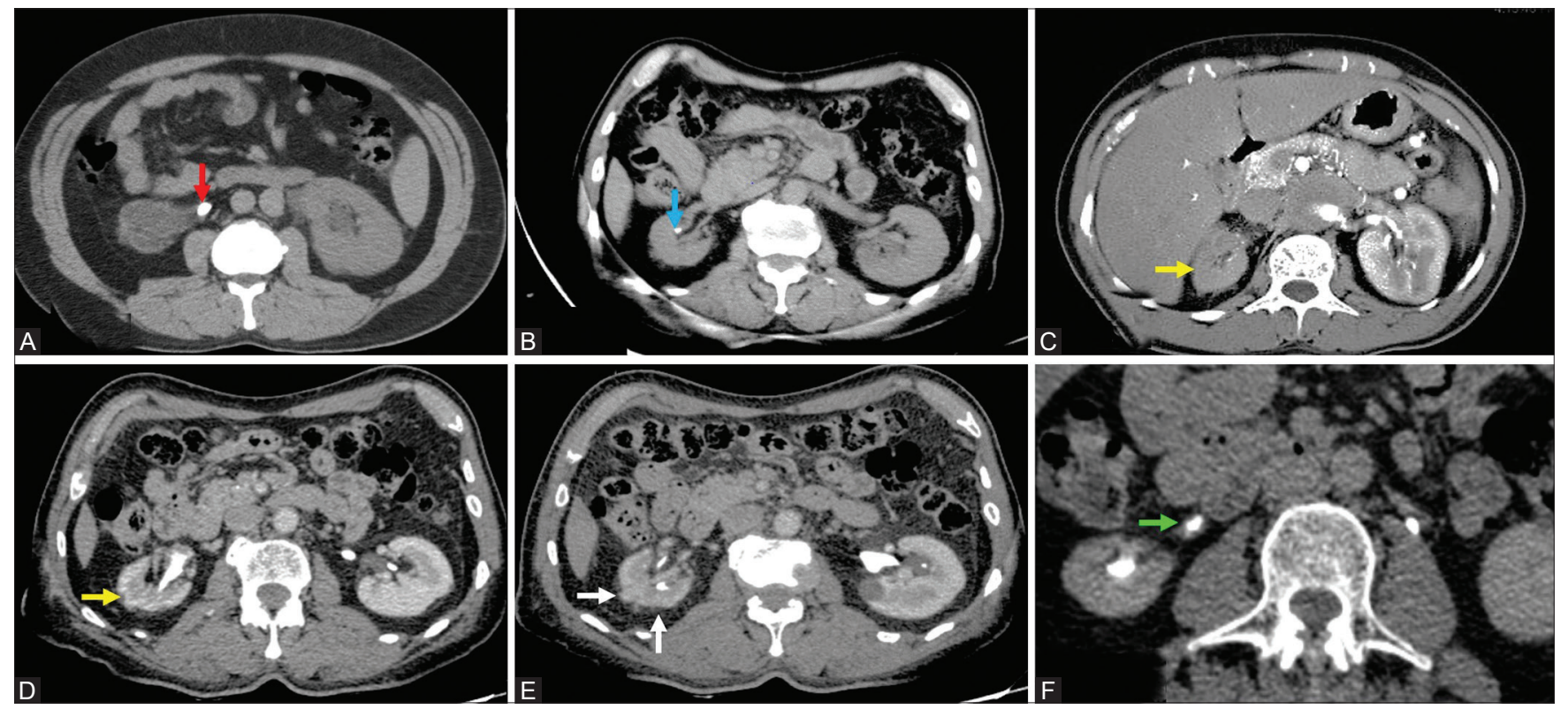

Figure 3 (A-F): CT urography in the same patient - (A) NCCT shows right PUJ calculus (red arrow); (B) NCCT shows nephrostomy tube in situ (blue arrow); (C) shows hypo-enhancement of the right kidney in nephrographic phase (yellow arrow); (D) shows hypo-enhancement of the right kidney in cortico-medullary phase (yellow arrow); (E) shows perinephric fat stranding around right kidney (white arrows); (F) shows excretion into the right ureter, ureter shows wall thickening and surrounding fat stranding (green arrow). 

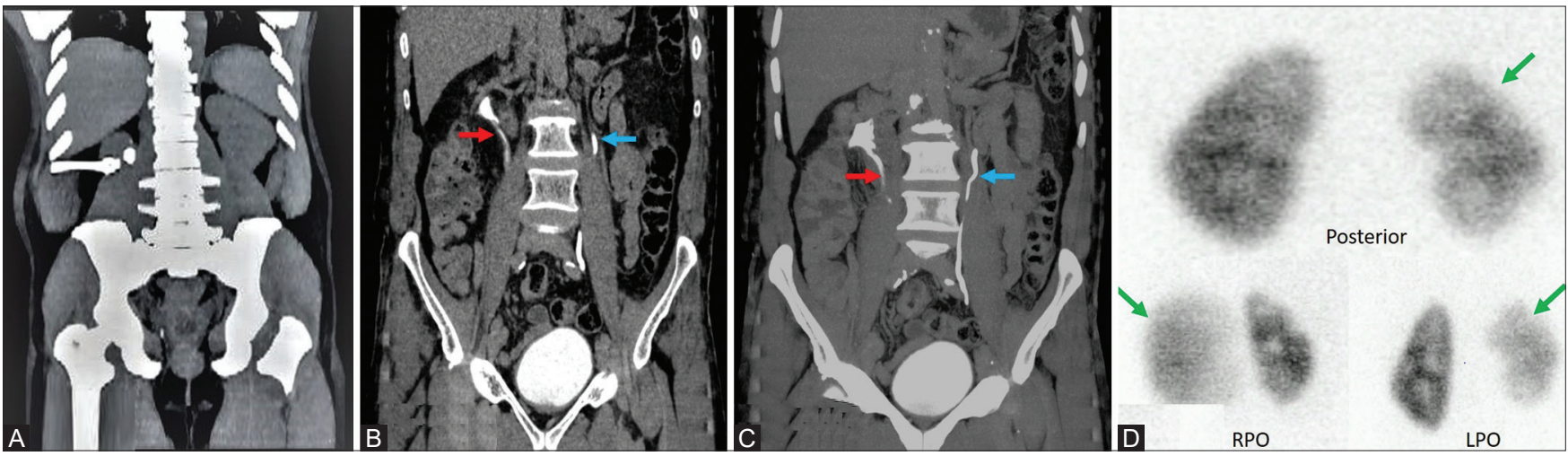

Figure 4 (A-D): Coronal MPR reconstruction of CT urography and late phase Tc99 DMSA examination in the same patient - (A) NCCT shows right PUJ calculus and nephrostomy tube; (B and C) excretory phase of CT urography shows good excretion in both the right (red arrows) and the left (blue arrows) ureters; (D) shows Tc99 DMSA scan which reveals delayed excretion and reduced size of right kidney, differential function found to be $30 \%$ on right side (green arrows).
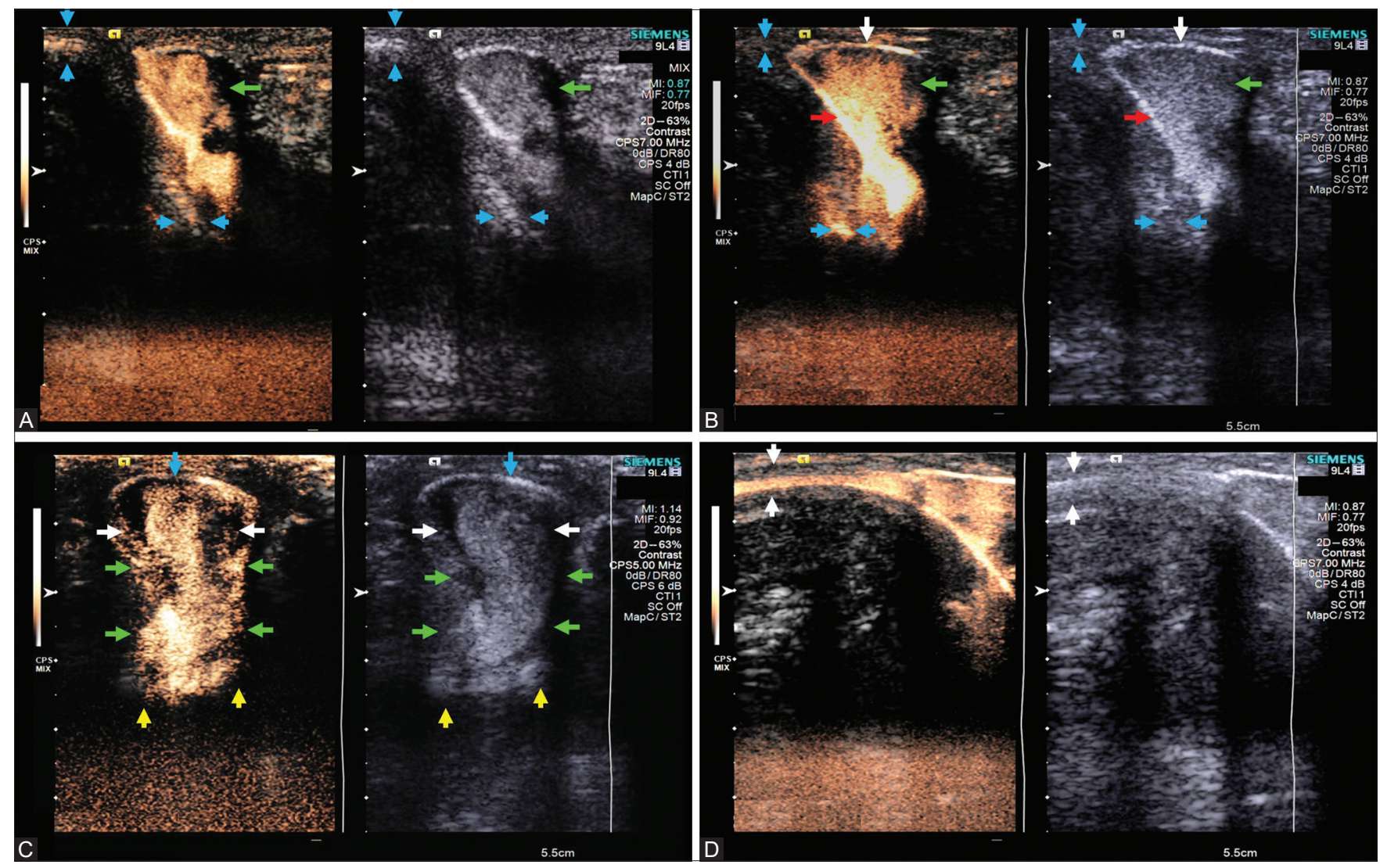

Figure 5 (A-D): CEVUS in the patient diagnosed with urethral strictures and diverticulum; (A) sagittal scan early voiding phase: peno-bulbar diverticulum (green arrow), normal corpus spongiosum (CS) (blue arrows); (B) late voiding phase: profuse filling of diverticulum (green arrow) \& enhancement of wall (red arrow), thinning of CS (white arrow), normal adjoining CS (blue arrows); (C) axial scan late voiding phase: normal urethral lumen (white arrows), diverticular neck and lumen (green arrows), thinning of CS with contrast leaking into surrounding soft tissues (yellow arrows); (D) normal CS (white arrows).

examination could delineate in dynamic mode, the two urethral strictures, and filling up of the large diverticulum located at the peno-bulbar junction. Additionally, the study revealed thinned-out corpus spongiosum with extension of disease into the adjoining soft tissues [Figure 5]. There was no evidence of VUR.
Two-stage urethroplasty for excision and reconstruction of the strictures and diverticulum was undertaken [Figure 6]. Six weeks later, right pyelo-lithotomy was performed with uneventful recovery. The right renal function subsequently improved over a period of 12 weeks, which was confirmed by Tc99 DMSA scans. Incidentally, the necessity of a right 


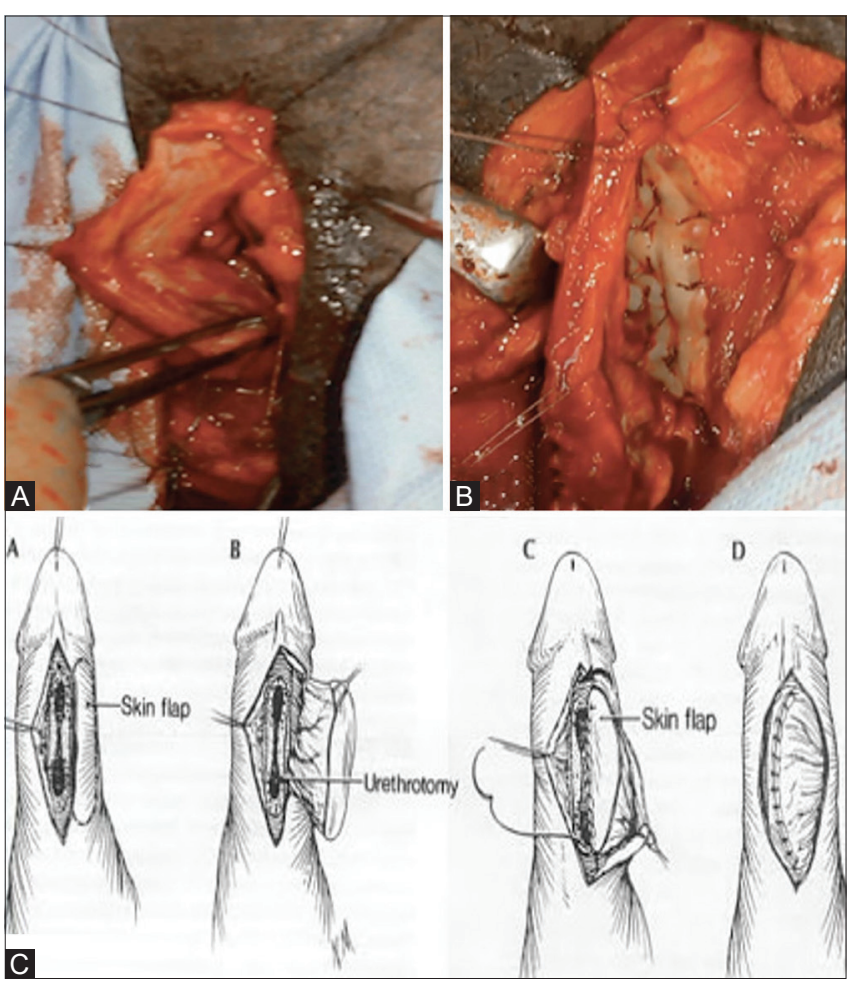

Figure 6 (A-C): Intraoperative photographs and diagram of surgical management in the 45-year-old male patient, diagnosed with right $P U J$ calculus, complicated by post-obstructive right renal atrophy, urethral strictures, and diverticulum - (A) excision of the diverticulum; (B) urethroplasty performed using buccal mucosa re-inforcement; (C) line diagram representing the surgical technique used.

nephrectomy advised by the previous medical institution was obviated.

\section{Discussion}

Male urethral diverticulum is an uncommon entity, more frequently of acquired origin and its exact prevalence remains unknown. ${ }^{[1-4,6]}$ The congenital variety is believed to occur due to developmental defects of the urethral folds, whereas the more common acquired variety (accounting for $67-90 \%)$ are secondary to traumatic insult. ${ }^{[1,3,4,6]}$ The commonest locations are peno-bulbar region followed by bulbar urethra. ${ }^{[4-6]}$ Our patient had a history of childhood perineal trauma and the urethral diverticulum was located at the peno-bulbar region.

Although patients are typically asymptomatic from primary disease, symptomatic male patients present with complications of lower urinary tract obstruction, infection, calculi, or a palpable, spontaneously decompressing peno-scrotal mass. ${ }^{[1-4,6]}$ Our patient, in addition to having intermittent urinary retention, presented with extensive upper urinary tract disease which had initially distracted from the diagnosis of urethral diverticulum. Although associated upper urinary tract involvement has occasionally been documented in patients with urethral diverticulum; nevertheless, no significant association between the upper and lower urinary tract pathology could be established in our patient.

Imaging evaluation remains essential for confirming the diagnosis, documenting the site, size, and surrounding pathology, in patients with urethral diverticulum. ${ }^{[1-5]}$ Although, traditionally, US is considered a supplementary technique modality for diagnostic evaluation of the urinary tract, very recent literature recommends CEVUS for investigation of VUR, urethral strictures, and diverticula in children. ${ }^{[8-10,12]}$ CEVUS has been recommended as the modality of choice for the urinary tract in children, not only for its capability of dynamic display and safe repeatability but also for preventing radiation injury. ${ }^{[10]}$ Wang et al., have recently recommended CEVUS, for the pre-operative evaluation in a series of female patients with urethral diverticula and emphasized its advantage of dynamic display. ${ }^{[11]}$ There are other distinct advantages of CEVUS, including wide availability of equipment and technical ease in performing the investigation. Additionally, being a radiation-free modality, it is safer for children and young adults. Furthermore, since second generation US contrast media are known to be almost allergy free, this distinct benefit has also been sufficiently emphasized in the context of its safe application for patients of all age groups, where in iodinated contrast agents may cause reactions even with intraluminal applications. ${ }^{[11]}$ However, the major disadvantage, especially for adults, is that, only a limited field can be evaluated at a time.

In our patient, imaging studies were undertaken with standard modalities relevant to the presenting complaints. Although the diagnosis established was right renal obstructive atrophy with urethral strictures and urethral diverticulum, nevertheless, the status of the soft tissues surrounding the diverticulum could not be ascertained. There was no evidence of extension of disease beyond urethral lumen in the RGU-VCUG study. CEVUS not only demonstrated the diverticulum with its continuity to urethral lumen, its site, and size, but also showed thinning of the corpora spongiosa and extension of pathology into the surrounding soft tissue, all in real-time dynamic display [Figure 5].

The treatment of urethral diverticulum is excision and urethroplasty. ${ }^{[1,4,13]}$ Our patient had excellent recovery, following this procedure.

\section{Conclusion}

In conclusion, ours is among the initial reports utilizing CEVUS for evaluation of male urethral diverticulum in an adult patient. Therefore, we propose that with further 
experience, it may be considered as a preferred diagnostic technique in urethral pathologies not only for women and children, but also for adult males.

\section{Declaration of patient consent}

The authors certify that they have obtained all appropriate patient consent forms. In the form the patient(s) has/have given his/her/their consent for his/her/their images and other clinical information to be reported in the journal. The patients understand that their names and initials will not be published and due efforts will be made to conceal their identity, but anonymity cannot be guaranteed.

Financial support and sponsorship

Nil.

\section{Conflicts of interest}

There are no conflicts of interest.

\section{References}

1. Thakur N, Sabale V, Mane D, Mullay A. Male urethral diverticulum uncommon entity: Our experience. Urology Annals 2016;8:478-482.

2. Tublin M, Borhani A, Furlan A, Heller M. Diagnostic Imaging: Genitourinary. $3^{\text {rd }}$-ed. Philadelphia: Elsevier; 2016. Chapter 7, Urethra. p. 438-441.

3. Trivedi VD, Salve SA, Dangle P, Navale A, Merchant S, Farooq M. Anterior urethral diverticulum: Report of two cases. Indian J Radiol Imaging 2003;13:441-442.
4. Cinman N, McAninch J, Glass A, Zaid U, Breyer B. Acquired male urethral diverticula: Presentation, diagnosis and management. J Urol 2012;188:1204-1208.

5. Li X, Sa Y, Jin C, Xu Y. Traumatic urethral diverticula: A rare malformation of the male urethra. Urol Int 2013;90:484-486.

6. Rimon U, Hertz M, Jonas P. Diverticula of the male urethra: A review of 61 cases. Urol Radiol 1992;14:49-55.

7. Pavlica P, Barozzi L, Menchi I. Imaging of male urethra. European radiology 2002;13:1583-1596.

8. Ntoulia A, Back S, Shellikeri S, Poznick L, Morgan T, Kerwood J, et al. Contrast-enhanced voiding urosonography (ceVUS) with the intravesical administration of the ultrasound contrast agent Optison ${ }^{\mathrm{TM}}$ for vesicoureteral reflux detection in children: A prospective clinical trial. Pediatr Radiol 2017;48:216-226.

9. Duran C, Valera A, Alguersuari A, Ballesteros E, Riera L, Martin C, et al. Voiding urosonography: The study of the urethra is no longer a limitation of the technique. Pediatr Radiol 2008;39:124-131.

10. Duran C, Beltrán V, González A, Gómez C, Riego J. Contrast-enhanced voiding urosonography for vesicoureteral reflux diagnosis in children. RadioGraphics 2017;37:1854-1869.

11. Wang X, Dou C, Yan Y, Song L, Hu B, Ying T. Preoperative transurethral contrast-enhanced ultrasonography in the diagnosis of female urethral diverticula. J Ultrasound Med 2018;37:2881-2889.

12. Zhang W, Cai B, Zhang X, Zhou J, Qiu L, Yi H. Contrast enhanced voiding urosonography with intravesical administration of ultrasound contrast agent for the diagnosis of pediatric vesicoureteral reflux. Exp Ther Med 2018;16:4546-4552.

13. Elliott $S$, Metro M, Mc Aninch J. Long-term follow-up of the ventrally placed buccal mucosa on-lay graft in bulbar urethral reconstruction. J Urol 2003;169:1754-1757. 